\section{Tumour microembolism presenting as "primary pulmonary hypertension"}

\author{
M Hibbert, S Braude
}

\begin{abstract}
Pulmonary tumour microembolism is a rare cause of pulmonary hypertension. A case of rapidly progressive pulmonary hypertension in a patient with a past history of breast carcinoma is presented. Despite active consideration and investigation for malignancy as a cause, correct diagnosis was only made at necropsy.
\end{abstract}

(Thorax 1997;52:1016-1017)

Keywords: pulmonary hypertension, tumour microemboli.

Tumour microembolism is a recognised but extremely uncommon cause of pulmonary hypertension. Even in dyspnoeic patients with known metastatic disease, a high index of suspicion is necessary to initiate appropriate investigations.

We present a case of severe and rapidly progressive pulmonary hypertension in a patient with a past history of mastectomy for breast carcinoma. Extensive investigations revealed no evidence of metastatic disease, nor a cause for pulmonary hypertension. The diagnosis of adenocarcinomatous microembolism was made only at necropsy. This case demonstrates the extreme difficulty of diagnosis and underlines the need to consider actively this entity in patients with cancer who become breathless.

\section{Case report}

A 71 year old woman presented with a two week history of dry cough and rapidly progressive dyspnoea, with one episode of minor haemoptysis. She reported no fevers, chest pain, or risk factors for venous thrombosis. Two years previously she had undergone right radical mastectomy for stage I breast carcinoma. All axillary nodes were free from tumour, and subsequent screening had demonstrated no evidence of local tumour recurrence or metastatic spread. There was no other relevant history.

On admission the patient was in respiratory distress (respiratory rate $28 / \mathrm{min}$ ) and was centrally cyanosed. The pulse was regular at 96 beats/min and she was peripherally well perfused with a normal blood pressure. Further examination revealed no signs of right heart strain or failure and the lung fields were clear. There was no evidence of deep venous throm- bosis. The examination was otherwise normal. Initial investigations revealed severe hypoxia $\left(\mathrm{PaO}_{2} 43.6 \mathrm{mmHg}(5.8 \mathrm{kPa}), \mathrm{PaCO}_{2} 28.7 \mathrm{mmHg}\right.$ $\left.(3.8 \mathrm{kPa}), \mathrm{FiO}_{2} 0.21\right)$ and mild elevation of serum alkaline phosphatase. Biochemistry, liver function tests, cardiac enzymes, and full blood profile were otherwise normal. The chest radiograph was normal. The electrocardiograph showed P pulmonale but no other features of right heart strain or hypertrophy. Echocardiography demonstrated moderate right atrial and right ventricular enlargement, with moderate tricuspid regurgitation and an estimated pulmonary artery systolic pressure of $60 \mathrm{mmHg}$. The left ventricular ejection fraction was normal. A ventilation:perfusion lung scan revealed a mild global reduction in perfusion with no segmental defects and normal ventilation. Pulmonary artery catheterisation confirmed severe pulmonary hypertension with a pulmonary artery pressure (PAP) of 67/ $15 \mathrm{mmHg}$ and a pulmonary vascular resistance (PVR) of 550 dyne s.cm ${ }^{-5}$. The patient was initially managed with oxygen therapy alone with marked symptomatic improvement.

Further investigations were then undertaken to define possible causes of the patient's pulmonary hypertension. Pulmonary angiography was normal with no evidence of embolic disease. Computed tomographic scans of the chest and abdomen (Siemens Somatom DR2 with $8 \mathrm{~mm}$ cuts) were also normal with no evidence of metastatic disease. These films were of good quality as the patient was able to breath-hold with supplemental oxygen. Fibreoptic bronchoscopic examination revealed no abnormalities. The patient declined transoesophageal echocardiography to exclude an intracardiac shunt. Serological screening for connective tissue disease was negative. In the absence of a precipitating cause a provisional diagnosis of primary pulmonary hypertension was made. An assessment of acute responsiveness to vasodilators ${ }^{1}$ demonstrated a $10 \%$ fall in pulmonary vascular resistance with no fall in pulmonary artery pressure and no significant systemic hypotension. The patient was commenced on coumarin and diltiazem and home oxygen therapy was arranged. She was discharged 10 days after admission.

After five days she presented again with worsening dyspnoea $\left(\mathrm{PaO}_{2} 55.3 \mathrm{mmHg}(7.4 \mathrm{kPa})\right.$, $\left.\mathrm{PaCO}_{2} 20.9 \mathrm{mmHg}(2.8 \mathrm{kPa}), \mathrm{FiO}_{2} 0.4\right)$ and gross signs of right heart failure. Initial treatment included oxygen therapy and diuretics, and resulted in little benefit. Repeat pulmonary artery catheterisation revealed a dramatic increase in pulmonary artery pressure to near systemic levels (PAP 76/45 mmHg, PVR 1400 dyne s. $\mathrm{cm}^{-5}$ ). Over the ensuing 24 hours the patient's oxygenation deteriorated further, requiring intubation and mechanical ventilation. Despite an infusion of prostacyclin, hypoxia worsened, accompanied by hypotension unresponsive to inotropes and the patient died three days after admission.

At necropsy both lungs macroscopically contained a few small nodules (maximum diameter $5 \mathrm{~mm}$ ) in the parenchyma and bronchial lymph nodes. The heart was normal. The liver showed 


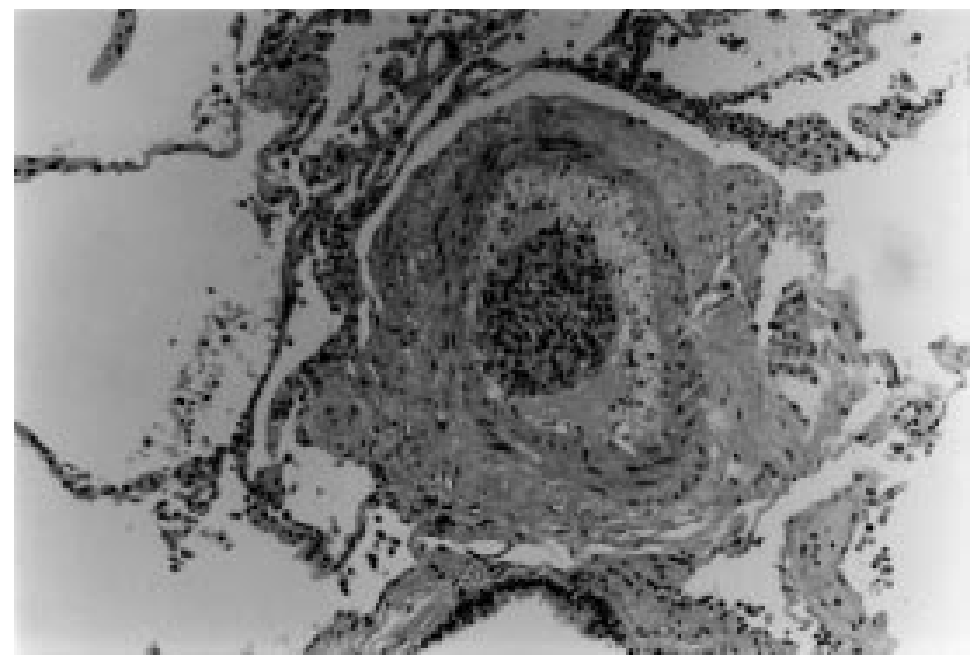

Figure 1 Tumour microembolus in a small pulmonary artery. Stain: haematoxylin and eosin; original magnification $\times 20$.

a nutmeg pattern of congestion. The spleen contained five nodules, the largest $8 \mathrm{~mm}$ in diameter, and the kidneys had a normal appearance. Microscopic examination revealed metastatic adenocarcinoma in the lungs, spleen, and renal capsule. The pulmonary microvasculature contained numerous tumour microemboli associated with intimal fibrosis in most of the small arterioles (fig 1). The site of origin was consistent with a primary breast adenocarcinoma. The necroscopic examination was otherwise normal.

\section{Discussion}

Pulmonary tumour microembolism itself is not uncommon, but is rarely recognised clinically. In $26 \%$ of 366 patients with malignancy, pulmonary tumour microemboli were noted at necropsy. ${ }^{2}$ In another large necropsy study, microscopic pulmonary emboli without any significant parenchymal metastases were seen in $2.4 \%$ of cases. ${ }^{3}$ The most frequent primaries involved were breast, liver, and choriocarcinoma. Tumour microembolism is, however, a rare cause of pulmonary arterial hypertension, with death from this cause requiring a majority of the pulmonary vasculature to be occluded. ${ }^{4}$ Prominent initial intimal hyperplasia, seen in association with tumour cells, is later followed by thrombus formation.
Unexplained dyspnoea, with or without signs of pulmonary hypertension, is the most frequent initial presentation. Radionuclide lung scanning in cases of diffuse intravascular metastatic tumour emboli is said to be characteristic with multiple small subsegmental perfusion defects and normal ventilation. ${ }^{5}$ Normal results have, however, been seen. ${ }^{67}$ Pulmonary angiography may be normal ${ }^{5}$ or may mimic primary pulmonary hypertension.

This case is of particular interest as the diagnosis of tumour microembolism was actively considered but was felt to be unlikely in view of the lack of clinical or imaging evidence of metastatic disease. Lung biopsy specimens (open or transbronchial) would have been required to make the diagnosis but were not felt to be warranted. Although correct antemortem diagnosis would have been unlikely to alter the outcome in this patient, future advances in anti-cancer therapy may lend more relevance to the recognition of this condition. The current mean survival from onset of dyspnoea still ranges from four to 12 weeks. $^{28}$ The course of this illness was notable also for the patient's rapid stepwise progression and final fulminant presentation. This presumably was caused by recurrent showers of tumour microemboli and progressive morphological changes in the pulmonary vascular bed.

This case demonstrates the extreme diagnostic difficulties in patients with pulmonary hypertension and tumour microembolism. It emphasises the need to consider this diagnosis actively in all patients with a history of malignancy who present with dyspnoea without an obvious cause.

1 Rich S, Kaufmann E, Levy PS. The effect of high doses of calcium-channel blockers on survival in primary pulmonary hypertension. N Engl F Med 1992;327:76-81.

2 Winterbauer RH, Elfenbein IB, Ball WC Jnr. Incidence and clinical significance of tumour embolisation to the lungs. Am 7 Med 1968;45:271-90

3 Kane RD, Hawkins HK, Miller JA, Noce PS. Microscopic pulmonary emboli associated with dyspnoea. Cancer 1975 36:1473-82.

4 Gorham LW. A study of pulmonary embolism II. The mechanism of death, based on clinicopathological investigation of 100 cases of massive and 285 cases of minor embolism of the pulmonary artery. Arch Intern Med 1961;108:189.

5 Sostman HD, Brown M, Toole A, Bobrow S, Gottschalk A. Perfusion scan in pulmonary vascular/lymphangitic carPerfusion scan in pulmonary vascular/lymphangitic carcinomatosis:

6 Scully RE, Galdabini JJ, McNeely BU. Case records of the Massachusetts General Hospital. N Engl F Med 1980;303: Massachuset.

7 Come PC. Echocardiographic recognition of pulmonary arterial disease and determination of its cause. $\mathrm{Am} 7 \mathrm{Med}$ terial disease and

8 Gonzales-Vitale JC, Garcia-Bunuel R. Pulmonary tumour emboli and cor pulmonale in primary carcinoma of the lung. Cancer 1976;38:2105-10. 